\title{
A complex role for the progesterone receptor in the response to vascular injury
}

\author{
Richard H. Karas, ${ }^{1}$ Martin van Eickels, ${ }^{1}$ John P. Lydon, ${ }^{2}$ Sean Roddy, ${ }^{3}$ Moon Kwoun, ${ }^{3}$ \\ Mark Aronovitz, ${ }^{1}$ Wendy E. Baur, ${ }^{1}$ Orla Conneely, ${ }^{2}$ Bert W. O’Malley, ${ }^{2}$ \\ and Michael E. Mendelsohn ${ }^{1}$
}

\begin{abstract}
${ }^{1}$ Molecular Cardiology Research Institute, New England Medical Center Hospitals Inc., Tufts University School of Medicine, Boston, Massachusetts, USA

${ }^{2}$ Baylor College of Medicine, Department of Cell Biology, Houston, Texas, USA

${ }^{3}$ Department of Vascular Surgery, New England Medical Center Hospitals Inc.,

Tufts University School of Medicine, Boston, Massachusetts, USA
\end{abstract}

Address correspondence to: Michael E. Mendelsohn, Molecular Cardiology Research Institute,

New England Medical Center Hospitals Inc., 750 Washington Street No. 080, Boston, Massachusetts 02111, USA.

Phone: (617) 636-9370; Fax: (617) 636-1444; E-mail: mmendelsohn@lifespan.org.

Received for publication September 21, 2000, and accepted in revised form June 18, 2001.

\begin{abstract}
Clinical studies of hormone replacement therapy to prevent cardiovascular diseases have heightened interest in the cardiovascular effects of progestins. However, the role of the progesterone receptor $(\mathrm{PR})$ in vascular biology has not been studied in vivo. We studied ovariectomized female PR knockout (PRKO) mice and their wild-type (WT) littermates using the mouse carotid artery injury model. Placebo-treated PRKO mice showed significantly greater vascular medial hypertrophy and vascular smooth muscle cell (VSMC) proliferation in response to vascular injury than did WT mice. Progesterone had no significant effect in the PRKO mice, but worsened the response to injury in WT mice. VSMCs cultured from PRKO mouse aortae were markedly hyperproliferative, and their growth was not affected by progesterone. In contrast to the in vivo findings, progesterone inhibited proliferation of WT-derived VSMCs. Furthermore, reintroduction of PR into PRKO-derived VSMCs using adenoviral methods restored progesterone-mediated inhibition of proliferation to these cells. This effect was reversed by the PR antagonist, RU 486. Thus, the effects of PR and progesterone differ markedly between cultured VSMCs and intact blood vessels. These data demonstrate a direct role for the PR in regulating the response to vascular injury and VSMC proliferation.
\end{abstract}

J. Clin. Invest. 108:611-618 (2001). DOI:10.1172/JCI200111374.

\section{Introduction}

Estrogen receptors are expressed in cardiovascular tissues, and the direct effects of estrogen on the cardiovascular system are now well recognized (reviewed in ref. 1,2). Much less is known, however, regarding the vascular expression of progesterone receptors and the effects of progestins on the cardiovascular system. The essential role of progestins in the behavioral and physiologic processes that maintain mammalian pregnancy have been recognized for many years $(3,4)$. More recently, progestins have also been shown to regulate many physiologic processes that impact on the atherosclerotic process. For example, progestins can adversely affect circulating levels of atherogenic and atheroprotective lipids (5) and expression of coagulation and thrombolytic proteins (6-8). Progestins also can inhibit vasorelaxation (9-12) and decrease proliferation of both vascular smooth muscle cells (VSMCs) (13-15) and endothelial cells $(15,16)$ in vitro. Although many observational studies, most of them related to primary prevention, support the atheroprotective effects of unopposed estrogens in postmenopausal women (reviewed in refs $1,2,17$ ), the vascular effects of hormone replacement therapy (HRT) with estrogens in combination with progestins remain controversial. Progestins have been reported in animal studies to reverse estrogen's atheroprotective effects in both nonhuman primate (18-20) and rat models (21-23). Earlier observational studies of women without known coronary artery disease (CAD) suggest that combined HRT was as beneficial as estrogen alone (24). In contrast, the Heart and Estrogen/Progestin Replacement Study (HERS), a largescale, randomized, controlled trial for secondary prevention demonstrated no benefit of treatment with combined HRT over 4.1 years in women with established CAD (25). Although many factors likely contributed to the neutral outcome in HERS (e.g., insufficient duration of follow up), some investigators have speculated that the neutral outcome in HERS resulted from the addition of a progestin, theorizing that its presence in this population might offset the beneficial vascular effects of estrogen alone.

Progesterone receptors (PRs), like other sex steroid hormone receptors, are members of a superfamily of ligandactivated transcription factors that regulate gene expres- 
sion following hormone binding (26-31). PRs are expressed in vascular cells $(13,32-34)$, and PR expression in vascular tissues is induced by estrogen $(32,33)$, supporting that direct vascular effects of progestins and/or estrogen may be mediated in part by PRs. To date, however, a direct role for PRs in vascular biology has not been studied in vivo. Therefore, we hypothesized that progesterone, acting via the $\mathrm{PR}$, can directly alter the response to vascular injury. To examine the role of progesterone and PRs in the vascular injury response in vivo, we used a mouse model of vascular injury (35-37) to examine the response to carotid arterial injury in female PR knockout (PRKO) mice and their wild-type (WT) littermates.

\section{Methods}

The PRKO mice were generated by targeted disruption of exon 1 of the PR gene and are devoid of both known forms of $\mathrm{PR}, \mathrm{PR}_{\mathrm{A}}$ and $\mathrm{PR}_{\mathrm{B}}$ (38). These mice develop normally into adulthood, though the female mice have selected reproductive (39) and endocrinologic (40) abnormalities.

Mouse model of carotid arterial injury. The mouse carotid injury model used in this study has been described in detail previously $(35-37,41)$. The experimental design of the present study is shown in Figure 1. Fifty-nine adult female mice (29 PRKO, 30 WT littermates) were ovariectomized, allowed to recover for 7-10 days, and then randomly assigned to receive vehicle or subcutaneous pellets that continuously release progesterone (35 mg) for 21 days (Innovative Research of America, Sarasota, Florida, USA). Previous studies have shown that ovariectomy reduces circulating progesterone to undetectable levels and that the progesterone pellets used in this study produce constant and physiologically relevant circulating hormone levels in mice (38-40, 42-44). One week after pellet implantation, anesthetized mice underwent unilateral common carotid artery injury by intraluminal passage of a fine wire, denuding the vascular endothelium and inducing a characteristic increase in medial area and VSMC proliferation. On the same day as the carotid injury, the mice were implanted subcutaneously with an osmotic minipump (Alzet Inc., Palo Alto, California, USA) that continuously releases bromo-deoxy-uridine (BrdU; 25 $\mathrm{mg} / \mathrm{kg} / \mathrm{d}$ ) throughout the experiment.

Morphometry and immunohistochemistry. Two weeks after carotid artery injury, vessels were harvested and the response to vascular injury was assessed using two independent measures: VSMC proliferation, measured by immunohistochemical detection of BrdUlabeled cells, and medial area, measured by computerized morphometry.

Parallel sections from all 118 carotid arteries were stained as described previously for hematoxylin/eosin and for elastin, and area measurements were made using a computerized morphometric analysis system on the elastin-stained sections (35-37). For each animal, two sections were analyzed, and the areas were averaged. Immunostaining also was performed on parallel carotid artery sections using anti-BrdU Ab's, endothelial cell-specific Ab's (factor VIII-related antigen), and VSMC-specific Ab's ( $\alpha$-actin) (all from Sigma Chemical Co., St. Louis, Missouri, USA) (35-37) to distinguish between different vascular cell types. Medial VSMCs that were labeled with BrdU were quantified as described (35-37). Adventitial cells were not included in any of these analyses. All analyses were performed by two observers blinded to mouse genotype and treatment group.

Construction of $P R$ adenoviruses. A full-length cDNA fragment coding for WT human $\mathrm{PR}_{\mathrm{B}}$ was cloned as a BamHI fragment (partial digest, to spare the internal BamHI site at position 70 of the human PR cDNA) from the plasmid $h \mathrm{PR}_{\mathrm{B}}$-pLEM into the adenovirus shuttle vector pACCMV.pLpA. The adenovirus adeno$\mathrm{PR}_{\mathrm{B}}$ was constructed by cotransfection of $\mathrm{PR}_{\mathrm{B}}-\mathrm{PAC}$ CMV.pLpA and pJM17 into HEK293 cells followed by standard selection and virus-purification procedures. Correct insertion of the appropriate cDNA into the shuttle vector was confirmed by sequence analysis.

Immunoblotting. To confirm proper expression of $\mathrm{PR}_{\mathrm{B}}, \mathrm{VSMCs}$ were infected with the adeno- $\mathrm{PR}_{\mathrm{B}}$ virus or a control adeno-green fluorescent protein (adenoGFP) virus, and 24 hours later the cells were lysed in the following buffer: $20 \mathrm{mM}$ Tris $\mathrm{HCl}(\mathrm{pH} 7.5), 140$ $\mathrm{mM} \mathrm{NaCl}, 2$ mM EDTA (pH 7.4), $50 \mathrm{mM}$ beta-glycerol phosphate, $1 \%$ (vol/vol) Triton X-100, 20\% (vol/vol) glycerol. Immediately before use, 1:1000 (vol/vol) of inhibitor cocktail set III was added to the lysis buffer (Calbiochem-Novabiochem Corp., San Diego, California, USA). Total cellular proteins $(25 \mu \mathrm{g})$ were separated by SDS-PAGE, transferred to nitrocellulose membranes, incubated with the primary $A b$, mouse monoclonal anti-PR Ab, Ab-8, at a dilution of 1:500 (NeoMarkers Inc., Fremont, California, USA) followed by the secondary Ab, anti-mouse IgG horseradish peroxidase-linked Ab (Amersham Pharmacia Biotech, Piscataway, New Jersey, USA), and developed with enhanced chemiluminescence techniques. Additional

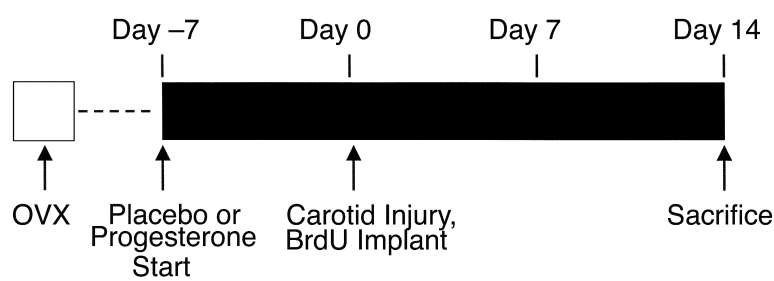

\section{Figure 1}

Design of the carotid artery injury study. All animals were ovariectomized (OVX) and then allowed to recover for 7-10 days. Progesterone-containing or placebo pellets were then implanted subcutaneously 1 week before the animals underwent unilateral carotid injury and implantation of an osmotic mini-pump containing BrdU. On day 14 after injury, the animals were sacrificed and both carotid arteries were perfusion fixed, harvested, and then embedded in paraffin. Subsequently, the vessels were sectioned transversely and stained to allow morphometric and immunohistochemical analysis of the response to injury. Further details of the procedure have been published previously (35-37). 


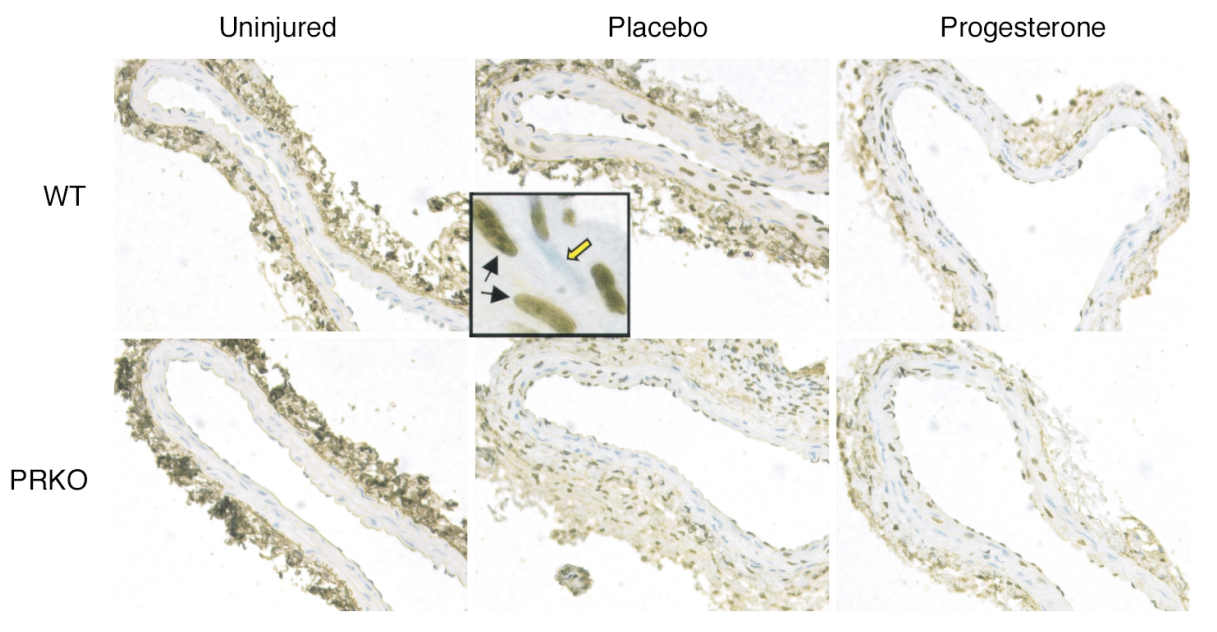

\section{Figure 2}

BrdU-stained sections of uninjured and injured WT and PRKO mouse carotid arteries. BrdU-stained carotid artery sections representative of the mean BrdU counts for each group are shown $(\times 200)$. Total BrdU counts were made on the complete section. In the inset, examples of both BrdUpositive VSMCs (black arrows) and BrdU-negative VSMCs (open yellow arrow) are shown $(\times 200)$. experiments were performed using monoclonal antiPR Ab's, Ab-7 and Ab-4 (Amersham Pharmacia Biotech), and the polyclonal anti-PR Ab C-19 (Santa Cruz Biotechnology Inc., Santa Cruz, California, USA).

Cell culture and proliferation assays. Aortic VSMCs were obtained by the explant method as described previously (45). Cultured cells were identified as VSMCs by immunostaining for smooth muscle-specific $\alpha$-actin according to the manufacturer's instructions (Sigma Chemical Co.). VSMCs were cultured from each of three individual PRKO and WT mice. VSMCs were grown in phenol red-free DMEM with $10 \%$ estrogendeficient FBS, as described (45).

VSMC proliferation was assessed under baseline conditions in the absence of mitogenic stimulation and in the presence of stimulation with FBS. Eight replicates of each cell type were included in each experiment, and each experiment was performed independently a minimum of three times. Proliferation assays included cells from each of the three cultures of VSMCs derived independently from different animals for each genotype, and the results were then pooled.

For the baseline VSMC proliferation assays, cells at passage 20 or less were plated in 96-well plates at a density of $5 \times 10^{3}$ cells/well. Six hours after plating, the cells were growth arrested for 16 hours in serum-free medium (SFM), at which time $0.1 \mu \mathrm{Cu} /$ well ${ }^{3} \mathrm{H}$-thymidine was added to each well. After 24 or 48 hours of incubation in SFM with ${ }^{3} \mathrm{H}$-thymidine, the cells were rinsed three times with ice-cold PBS and then treated with 5\% trichloroacetic acid for 20 minutes at $4^{\circ} \mathrm{C}$, followed by the addition of an equal volume of $0.5 \mathrm{~N} \mathrm{NaOH}$. Finally, the cell lysate was neutralized by addition of $0.5 \mathrm{~N} \mathrm{HCl}$, mixed with scintillation fluid, and the magnitude of ${ }^{3} \mathrm{H}$ thymidine uptake determined by scintillation counting. To examine the effect of progesterone on proliferation of VSMCs stimulated with mitogens, WT- and PRKOderived VSMCs were plated at a density of $20 \times 10^{3}$ cells/well and incubated in SFM in the presence or absence of progesterone and/or the PR antagonist RU 486. RU 486 was added to the appropriate wells 1 hour before the addition of progesterone. All wells were treated with the same concentration of the vehicle ethanol. After allowing 24 hours for the cells to become quiescent, they were stimulated with $1 \% \mathrm{FBS}$, incubated with ${ }^{3} \mathrm{H}-$ thymidine for 24 hours, and the magnitude of ${ }^{3} \mathrm{H}$-thymidine uptake was then determined as described above. In a subset of experiments, the PRKO-derived VSMCs were incubated with either adeno- $\mathrm{PR}_{\mathrm{B}}$ or the control adenoGFP virus at moi's ranging from 1-50 for 24 hours before plating. Thereafter the cells were placed in SFM with or without progesterone and/or RU 486, stimulated with $1 \%$ FBS, and ${ }^{3} \mathrm{H}$-thymidine uptake was determined as described above. Total cell number was also determined in parallel experiments by manual counting on a hemocytometer 6 hours after plating to ensure equal plating of WT and PRKO VSMCs.

Statistical analyses. For all statistical analyses, data were first subjected to tests for normality and equal variance between groups. Genotype and treatment groups were then compared using a two-way ANOVA. Where significant findings were noted, subsequent post hoc analyses were carried out pairwise using the method of Student-Newman-Keuls. A $P$ value less than or equal to 0.05 was considered significant.

\section{Results}

Representative BrdU-stained sections are shown in Figure 2, and representative elastin-stained sections are shown in Figure 3. As expected, BrdU-labeled VSMCs were detected only rarely in the uninjured vessels of animals in all groups studied (mean $<1.0$ BrdU-positive cell/section, without significant differences between any treatment or genotype group). Uninjured carotid arteries from the WT and PRKO mice were indistinguishable morphologically, regardless of treatment assignment, and thus, as in our previous studies, the medial areas of the two groups were combined $\left(\right.$ mean $\left.=18.1 \pm 0.3 \times 10^{-3} \mathrm{~mm}^{2}\right)$.

Deletion of the PR increases the vascular injury response in placebo-treated female mice. Medial and intimal VSMC proliferation increased significantly in the injured carotid 


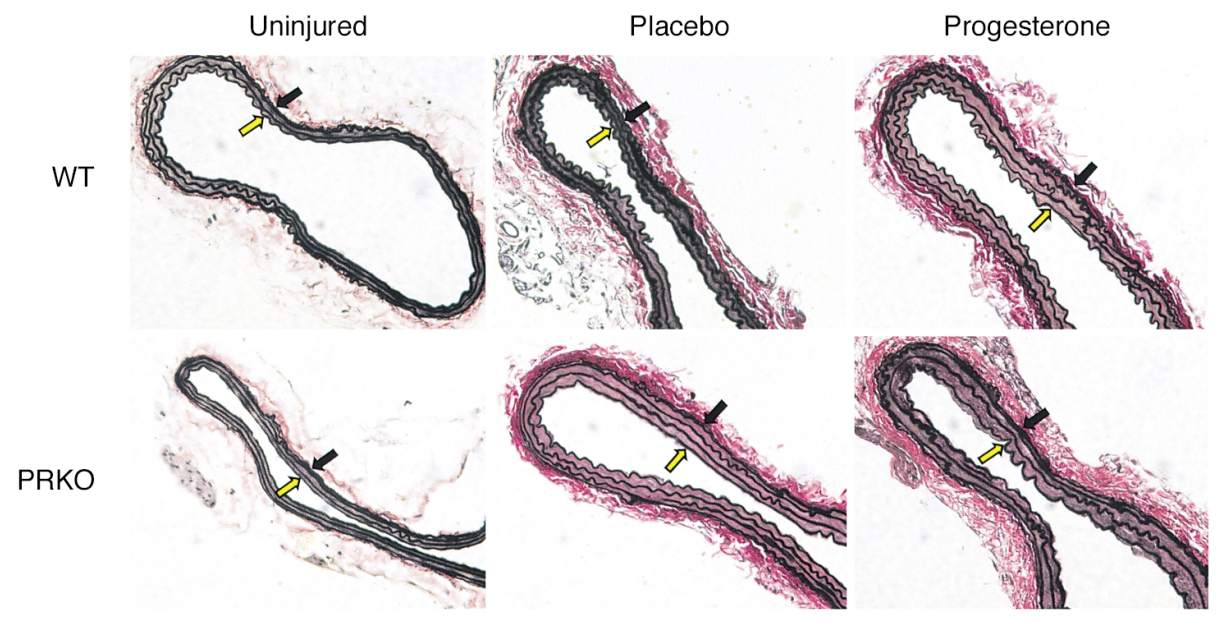

\begin{abstract}
Figure 3
Elastin-stained sections of uninjured and injured WT and PRKO mouse carotid arteries. Elastinstained carotid artery sections representative of the mean medial area for each group are shown $(\times 200)$. The media is delimited by the internal elastic lamina (open yellow arrow) and the external elastic lamina (black arrow).
\end{abstract}

artery in the placebo-treated WT mice $(23 \pm 4$ BrdUlabeled cells per section; $P<0.001$ vs. uninjured) and in the placebo-treated PRKO mice $(42 \pm 8$ BrdU-labeled cells per section; $P<0.001$ vs. uninjured). The injuryinduced increase in VSMC proliferation was significantly greater in the placebo-treated PRKO mice than in their WT littermates $(P<0.02)$. Injury also induced an increase in medial area in the WT placebo-treated mice $(20.6 \pm 1.0$ $\times 10^{-3} \mathrm{~mm}^{2} ; P<0.02$ vs. uninjured), and in the placebotreated PRKO mice $\left(24.1 \pm 1.4 \times 10^{-3} \mathrm{~mm}^{2} ; P<0.001\right.$ vs. uninjured). As with the BrdU labeling, the increase in medial area in the placebo-treated PRKO mice also was significantly greater than that seen in the placebo-treated WT littermates $(P<0.01)$. Thus, in the absence of endogenous or exogenous progesterone, disruption of PR expression resulted in an increase in both measures of the response to vascular injury.

Progesterone enhances the response to vascular injury in WT, but not PRKO female mice. BrdU-labeled VSMCs in the media and intima of the WT mice increased from $23 \pm$ 4 BrdU-positive cells per section in the placebo-treated animals to $40 \pm 6 \mathrm{BrdU}$-positive cells per section in the progesterone-treated group $(P<0.05)$. In the PRKO mice, however, the substantial BrdU labeling evident in the placebo-treated PRKO mice $(42 \pm 8 \mathrm{BrdU}$-positive cells per section) tended to be reduced by progesterone treatment ( $32 \pm 7$ BrdU-labeled cells per section), but this trend did not achieve statistical significance $(P=0.4)$. In WT mice, the increase in medial area fol-

\section{Figure 4}

VSMCs derived from female PRKO mice are hyperproliferative. Aortic VSMCs derived from female PRKO mice or their WT littermates were maintained in SFM and their rate of proliferation was assessed by ${ }^{3} \mathrm{H}$-thymidine uptake. Unlike the WT-derived cells, the PRKOderived cells did not become quiescent, but rather continued to proliferate markedly for at least 48 hours $\left({ }^{3} \mathrm{H}\right.$-thymidine uptake $10.1 \pm$ 1.6-fold and $13.1 \pm 1.8$-fold greater in PRKO vs. WT cells on day 1 and day 2 , respectively; $n=5$ independent experiments; ${ }^{*} P<0.001$ vs. WT for each day). lowing injury also was greater with progesterone treatment $\left(23.3 \pm 1.210^{-3} \mathrm{~mm}^{2}\right.$ in the progesterone-treated mice vs. $20.6 \pm 1.0 \times 10^{-3} \mathrm{~mm}^{2}$ in the placebo-treated mice; $P=0.01$ ). In contrast, in the placebo-treated PRKO mice the large increase in medial area following injury $\left(24.1 \pm 1.4 \times 10^{-3} \mathrm{~mm}^{2}\right)$ was somewhat attenuated by progesterone treatment $\left(21.4 \pm 1.3 \times 10^{-3} \mathrm{~mm}^{2}\right)$, though this trend was not statistically significant $(P=0.13)$. These data demonstrate that progesterone adversely affects the vascular injury response in WT animals, but does not significantly alter the already robust response to vascular injury in the PRKO mice.

VSMCs cultured from PRKO mice are hyperproliferative. To determine whether the increase in VSMC proliferation observed in vivo in the PRKO mice resulted from an intrinsic alteration in the VSMCs, a series of in vitro VSMC proliferation assays were performed. VSMC cultured from WT female mouse aortae and maintained in SFM remained quiescent over 24-48 hours as expected (Figure 4). In contrast, VSMCs derived from the female PRKO mice continued to proliferate throughout both day $1(10.0 \pm 1.6$-fold greater than WT-derived VSMCs) and day $2(13.1 \pm 1.8$-fold greater than the WT-derived VSMCs) of the experiment (Figure $4 ; n=5$ separate experiments, $P<0.0001$ vs. WT for each day), despite being maintained in SFM. In parallel control studies using VSMCs derived from male animals, both WT and PRKO-derived cells remained quiescent under the same serum-free conditions (data not shown).

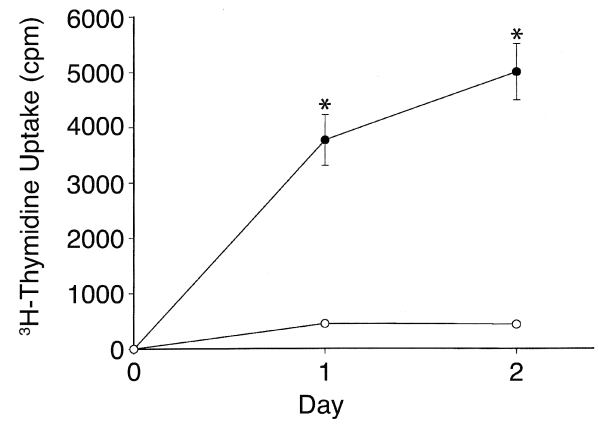




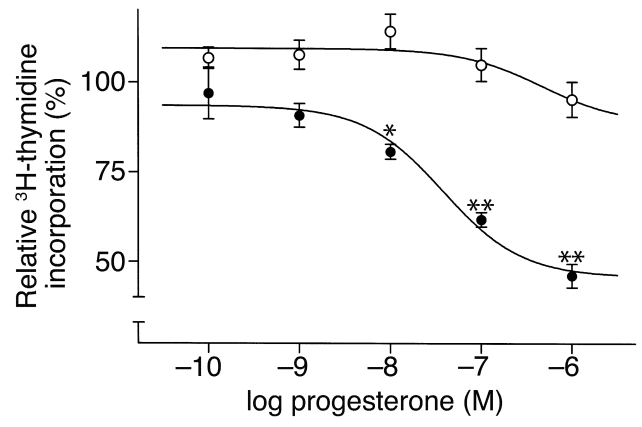

Progesterone inhibits proliferation of cultured VSMCs in a PRdependent manner. The effect of progesterone on FBSinduced proliferation of isolated, cultured VSMCs was examined next. Quiescent VSMCs cultured from WT or PRKO mice were stimulated with $1 \%$ FBS in the presence or absence of progesterone. Progesterone significantly inhibited FBS-induced growth of WT-derived VSMCs in a dose-dependent manner (Figure 5; maximum inhibition $54 \% \pm 3 \% ; \mathrm{IC}_{50}=34 \mathrm{nM}$ ), but had no significant effect on proliferation in PRKO-derived VSMCs (Figure 5). To investigate the role of the PR in mediating the antiproliferative effect of progesterone, PRKO-derived VSMCs were infected with various concentrations of adenovirus coding for expression of full-length, human $\mathrm{WT} \mathrm{PR}_{\mathrm{B}}$. Immunostaining for PR using a PR-specific Ab demonstrated that more than $95 \%$ of the VSMCs were successfully infected at an moi of 50 (data not shown). In the absence of progesterone, infection with either adeno- $P R_{B}$ or a control adenovirus encoding GFP had no significant effect on VSMC growth (data not shown). Furthermore, progesterone had no effect on proliferation of the GFPinfected PRKO VSMCs (data not shown). In contrast, progesterone dose-dependently inhibited growth of the

\footnotetext{
Figure 6

Progesterone-mediated inhibition of cultured VSMCs is mediated by the PR. (a) PRKO-derived VSMCs were infected with adeno-PR $R_{B}$ at moi's ranging from 1 to 50 , and the effect of progesterone (Prog) on FBS-stimulated ${ }^{3} \mathrm{H}$-thymidine uptake was examined. Progesterone inhibited VSMC proliferation with the greatest effect at an moi of 50 for adeno-PR . (Moi $=1$, black bars; moi $=10$, gray bars; and moi $=50$, open bars; ${ }^{*} P<0.01$ vs. moi $1,{ }^{*} P<0.001$ vs. moi 1 , and $P<0.01$ vs. moi 10). The inset depicts an immunoblot analysis demonstrating the expression of $\mathrm{PR}_{\mathrm{B}}$ in adeno- $\mathrm{PR}_{\mathrm{B}}$-infected $\mathrm{PRKO}$ cells $(\mathrm{moi}=50)$, while no expression was observed in adenoGFP-infected PRKO VSMCs. The positive control (hBCa) was provided by the manufacturer. (b) After infection of PRKO-derived VSMCs with an moi of 50 for adeno-PR $\mathrm{R}_{\mathrm{B}}$, progesterone dose-dependently inhibited growth of these cells, and progesterone-mediated growth inhibition was abolished by coincubation with the PR antagonist RU $486\left({ }^{*} P<0.01,{ }^{*} P<0.001\right.$ vs. control cells treated with vehicle alone). In $\mathbf{a}$ and $\mathbf{b}$, bars represent the mean \pm SE of each of three independent experiments each performed with eight replicates per condition. For each cell type, the results are shown relative to the ${ }^{3} \mathrm{H}$-thymidine uptake in the vehicle-treated cells as the absolute rate of uptake was significantly higher in the PRKO-derived cells compared with the WT-derived cells (compare Figure 4).
}

\section{Figure 5}

Progesterone inhibits proliferation of cultured VSMCs. Quiescent aortic VSMCs derived from female PRKO mice or their WT littermates were stimulated with $1 \%$ FBS in the presence or absence of progesterone, and their rate of proliferation was assessed by ${ }^{3} \mathrm{H}$ thymidine uptake. Progesterone treatment inhibited proliferation of WT-derived VSMCs (closed circles), but had no effect on proliferation of PRKO-derived VSMCs (open circles) $\left({ }^{*} P<0.05,{ }^{*} P<0.01\right.$ vs. control cells treated with vehicle alone). Shown are the mean \pm SE from three independent experiments, each performed with eight replicates for each concentration of progesterone.

$\mathrm{PR}_{\mathrm{B}}$-infected PRKO-derived VSMCs with the greatest effect observed at the moi of 50 of adeno- $\mathrm{PR}_{\mathrm{B}}$ (Figure 6a). Lack of PR protein in the PRKO-derived cells and expression of $\mathrm{PR}$ protein in the $\mathrm{PR}_{\mathrm{B}}$-infected cells (moi 50) was confirmed by Western blot analysis (Figure 6a, inset). To explore further the role of the PR in progesterone-mediated VSMC growth inhibition, the effects of the PR antagonist RU 486 were examined. Using an adeno- $\mathrm{PR}_{\mathrm{B}}$ moi of 50, progesterone dose-dependently inhibited thymidine uptake with a maximum inhibition of $58 \% \pm$ $6 \%$ and an $\mathrm{IC}_{50}=11 \mathrm{nM}$; Figure $6 \mathrm{~b}$ ). Coincubation of progesterone-treated cells with the PR antagonist RU 486 led to a dose-dependent reversal of the progesteronemediated growth inhibition (Figure 6b).

\section{Discussion}

In vivo findings. The findings from both in vivo measures of vascular injury used in these studies are consistent with each other and demonstrate previously unrecog-

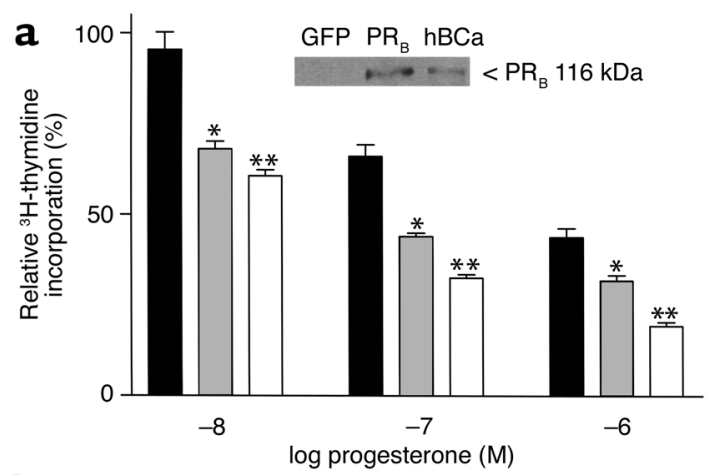

b

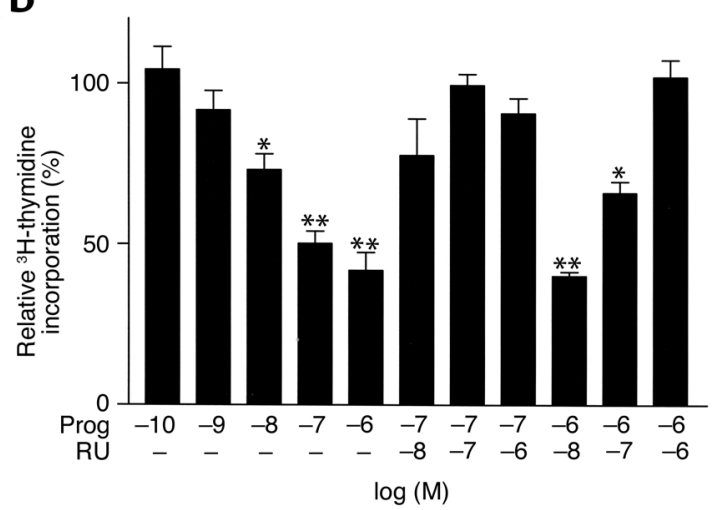




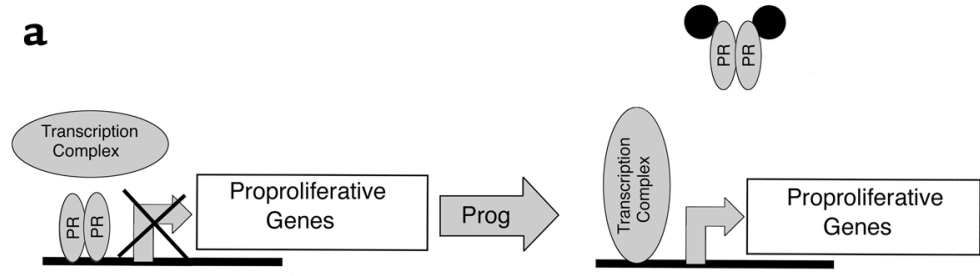

b

b

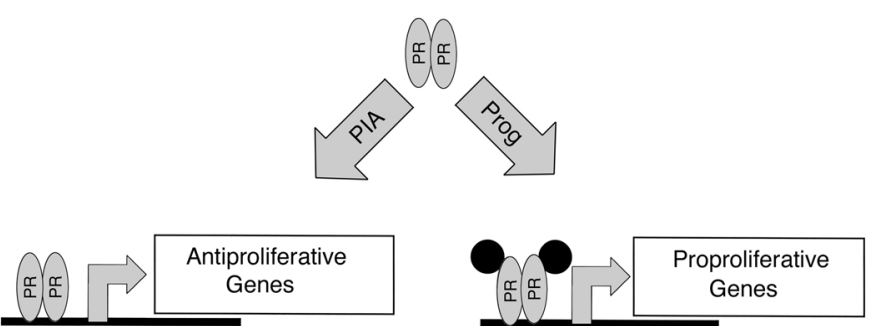

Figure 7

Two models of molecular mechanisms that may mediate PR/progesterone effects on the vascular injury response. For explanation, see text. Prog, progesterone; PIA, progesterone-independent activation.

nized functions of the PR in the cardiovascular system. The response to carotid artery injury was significantly greater in the PRKO mice than in the WT animals, and progesterone worsened the response to vascular injury in the WT mice, but had no significant effect on vascular injury in the PRKO mice. These data support a complex role for the PR in the response to vascular injury and in vascular biology. To our knowledge, this represents the first study directly implicating the PR in the vascular injury response in vivo.

The increased response to vascular injury in PRKO mice is likely multifactorial. The increased VSMC proliferation in the female PRKO mice following vascular injury suggests that the PR exerts an antiproliferative effect in VSMCs in vivo in WT animals. This is supported further by the in vitro VSMC proliferation assays demonstrating that female PRKO-derived VSMCs proliferate more rapidly than do WT-derived VSMCs, even in SFM. Thus, loss of PR expression and the resultant increase in VSMC proliferation is likely one mechanism that contributes to the increased vascular injury response in the PRKO mice. Interestingly, the hyperproliferative effect of PR disruption is observed in the absence of progesterone both in vivo and in vitro (see below). An important role for the PR in the cardiovascular system also is supported by the observation that progesterone-induced increases in the vascular injury response are observed in the WT but not the PRKO mice. It is interesting to note that progesterone tended to attenuate the response to injury in the PRKO mice, although this trend did not reach statistical significance. It is unclear whether this reflects a biologically relevant effect of progesterone that is PR-independent or whether it was indeed due to chance. The effects of progesterone on the vascular injury response have not been examined previously in mice. Earlier studies in other animal models regard- ing the effects of progestins on vascular injury have yielded conflicting results (18-23), likely due to differences in the dose and/or form of progestin used.

Several other mechanisms also could contribute to the increased response to vascular injury with progesterone treatment observed in the WT animals. Endothelial cells express PR, and progestins can inhibit endothelial cell migration and proliferation in vitro and in vivo (16). This is potentially an important mechanism in the mouse carotid artery injury model because restoration of an intact endothelium returns the underlying VSMCs to a quiescent state (reviewed in refs. 46, 47). Delayed re-endothelialization would thus prolong the period during which VMSCs proliferate. Additional effects of progestins on the endothelium include alterations in the production of tissue plasminogen activator- 1 and plasminogen activator inhibitor-1 (8), inhibition of endothelin-induced angiotensin II production (48), and enhancement of thrombospondin production (7), all effects that would be expected to affect the response to vascular injury.

In vitro findings. Cultured VSMCs have been shown to express PR (13-15), but little is known regarding the effects of progestins on VSMCs. In the current study, progesterone inhibited proliferation in VSMCs cultured from female WT mice, extending previous reports demonstrating that progesterone inhibits in vitro proliferation of aortic VSMCs derived from male rats (13). A role for the PR in mediating the antiproliferative effects of progesterone is supported by the observation that progesterone inhibits proliferation of PRKO-derived VSMCs infected with PR, but has no effect on proliferation in control (GFP-infected) PRKO-derived VSMCs. The reversal of progesterone's antiproliferative effect by the PR antagonist RU 486 observed in the PR-infected PRKO cells also supports this conclusion. These observations are limited to $P_{B}$ because this was the isoform of PR used in the experiments presented above. Further investigation of potential PR isoform-specific effects on VSMC proliferation is the subject of ongoing investigation.

Comparison of in vivo and in vitro findings. The antiproliferative effects of progesterone observed in VSMCs in vitro contrasts with the increased VSMC proliferation seen in vivo following injury in the progesterone-treated WT mice. This may result from several factors. The effect of progesterone on VSMCs may be altered, for example, by the presence and proximity of other cell types in vivo. In addition, the endpoint used to assess VSMC proliferation in the carotid injury model, BrdUlabeling of VSMCs over 2 weeks following injury, is a measure of the extent of VSMC proliferation, but not the rate of VSMC proliferation. The extent of VSMC 
proliferation is likely determined by several factors, only one of which is the rate of proliferation. For example, if progesterone directly reduces the rate of VSMC and endothelial cell proliferation, but inhibits endothelial cell proliferation to a greater degree, the net result might be to sufficiently delay re-endothelialization and extend the period during which VSMCs proliferate, thus increasing the overall extent of BrdUlabeled VSMCs. Further investigation of the differing effects of progesterone in vivo and in vitro will require additional experimentation, including time-course and coculture experiments using different vascular cell types. These differences highlight the importance of using in vivo, whole animal models to address the mechanisms that mediate hormonal effects on vascular cell growth and vascular injury.

Taken together, these findings implicate the PR in vascular biology and raise an intriguing mechanistic question: how does the loss of PR cause an increase in the vascular injury response, while progesterone treatment of WT animals augments the response to injury? Since the $P R$ is a transcription factor, differential effects of PR on the expression of specific vascular genes likely are involved. One model that could explain these findings is that the unliganded PR represses expression of proproliferative genes, and this repression is relieved upon hormone stimulation (Figure 7a). Though transcriptional repression such as this has been reported previously for the thyroid hormone receptor (another member of the superfamily of ligand-activated transcription factors; refs. 49, 50), PR-mediated transcriptional repression has only recently been observed (K. Horowitz, personal communication). An alternative model by which the PR could protect against vascular injury in the absence of progesterone, but worsen injury in the presence of progesterone, is shown in Figure 7b. In this model, PR activates vascular protective genes in the absence of progesterone, but upon hormone stimulation, an alternate set of proproliferative genes are transcribed that promote vascular injury. Hormone-independent activation of the PR and other steroid hormone receptors has been reported to occur via growth factor signaling pathways in both nonvascular and vascular cells (51-60). Thus, progesteronedependent and progesterone-independent activation of the PR could result in activation of different sets of genes that either inhibit or augment the vascular injury response. Alternatively, endogenous progesterone-like ligands may exist that are capable of activating the PR in the absence of progesterone, though such endogenous ligands have not been reported to date. Determination of the extent to which the pathways described in this model are unique to VSMCs or apply to other cell types will require additional investigation.

In summary, the data presented above demonstrate a direct role for the PR in the response to vascular injury. In the absence of progesterone, loss of the PR leads to more pronounced vascular injury, whereas in animals with an intact $\mathrm{PR}$, progesterone increases the vascular injury response. These data support that the PR regu- lates the vascular injury response, but it does so in a complex way, having the capacity to either augment or attenuate the degree of injury. These studies support that further understanding of the mechanisms that mediate the complex effects of PR on the vasculature may have important implications for current and newer hormone replacement therapies.

\section{Acknowledgments}

The authors thank Kathy Dunlap, Alan Kopin, and Simon Michael for helpful discussions regarding this manuscript. We also thank Sharon Lynch and Patricia Griffiths for expert preparation of this manuscript. This work was supported in part by NIH HL-61298 (R.H. Karas), NIH HL-59953 and NIH HL-56069 (M.E. Mendelsohn), HD-07857 (B.W. O'Malley), and CA77530-01 (J.P. Lydon). M. van Eickels was supported by a grant from Deutsche Forschungsgemeinschaft. These studies were performed during the time M.E. Mendelsohn was an Established Investigator of the American Heart Association.

1. Mendelsohn, M.E., and Karas, R.H. 1999. Mechanisms of disease: the protective effects of estrogen on the cardiovascular system. N. Engl. J. Med. 340:1801-1811.

2. Farhat, M.Y., Lavigne, M.C., and Ramwell, P.W. 1996. The vascular protective effects of estrogen. FASEB J. 10:615-624.

3. Baulieu, E.E. 1989. Contragestion and other clinical applications of RU486, an antiprogesterone at the receptor. Science. 245:1351-1357.

4. Clarke, C.L., and Sutherland, R.L. 1990. Progestin regulation of cellular proliferation. Endocr. Rev. 11:266-300.

5. 1995. Effects of estrogen or estrogen/progestin regimens on heart disease risk factors in postmenopausal women: the Postmenopausal Estrogen/Progestin Interventions (PEPI) Trial. The Writing Group for the PEPI Trial. JAMA. 273:199-208.

6. Nabulsi, A.A., et al. 1993. Association of hormone-replacement therapy with various cardiovascular risk factors in postmenopausal women. $N$. Engl. J. Med. 328:1069-1075.

7. Iruela-Arispe, M.L., Porter, P., Bornstein, P., and Sage, E.H. 1996. Thrombospondin-1, an inhibitor of angiogenesis, is regulated by progesterone in the human endometrium. J. Clin. Invest. 97:403-412.

8. Miyauchi, A., et al. 1995. Regulation of the plasminogen activator/plasmin system by epidermal growth factor in cultured human endometrial cells. Hum. Reprod. 10:3284-3288.

9. Mercuro, G., et al. 1999. Effects of acute administration of natural progesterone on peripheral vascular responsiveness in healthy postmenopausal women. Am. J. Cardiol. 84:214-218.

10. Miller, V.M., and Vanhoutte, P.M. 1991. Progesterone and modulation of endothelium-dependent responses in canine coronary arteries. Am.J. Physiol. 261:R1022-R1027.

11. Miller, V.T., et al. 1991. Effects of conjugated equine estrogen with and without three different progestogens on lipoproteins, high-density lipoprotein subfractions, and apolipoprotein A-I. Obstet. Gynecol. 77:235-240.

12. White, M.M., et al. 1995. Estrogen, progesterone, and vascular reactivity: potential cellular mechanisms. Endocr. Rev. 16:739-751.

13. Lee, W.S., Harder, J.A., Yoshizumi, M., Lee, M.E., and Haber, E. 1997. Progesterone inhibits arterial smooth muscle cell proliferation. Nat. Med. 3:1005-1008.

14. Morey, A.K., et al. 1997. Estrogen and progesterone inhibit vascular smooth muscle proliferation. Endocrinology. 138:3330-3339.

15. Okada, M., et al. 1997. Effects of $17 \beta$-estradiol and progesterone on migration of human monocytic THP-1 cells stimulated by minimally oxidized low-density lipoprotein in vitro. Cardiovasc. Res. 34:529-535.

16. Vazquez, F., et al. 1999. Progesterone regulates proliferation of endothelial cells. J. Biol. Chem. 274:2185-2192.

17. Grady, D., et al. 1992. Hormone therapy to prevent disease and prolong life in postmenopausal women. Ann. Intern. Med. 117:1016-1037.

18. Williams, J.K., Honore, E.K., Washburn, S.A., and Clarkson, T.B. 1994. Effects of hormone replacement therapy on reactivity of atherosclerotic coronary arteries in cynomolgus monkeys. J. Am. Coll. Cardiol. 24:1757-1761.

19. Clarkson, T.B. 1998. Effects of estrogens, progestins, and androgens on coronary vasomotion and atherosclerosis. J. Reprod. Med. 43:741-745. 
20. Clarkson, T.B., et al. 1990. Oral contraceptives and coronary artery atherosclerosis of cynomolgus monkeys. Am. J. Obstet. Gynecol. 75:217-222.

21. Levine, R.L., Chen, S.J., Durand, J., Chen, Y.F., and Oparil, S. 1996. Medroxyprogesterone attenuates estrogen-mediated inhibition of neointima formation after balloon injury of the rat carotid artery. Circulation. 94:2221-2227.

22. Oparil, S., Levine, R.L., Chen, S.-J., Durand, J., and Chen, Y.F. 1997. Sexually dimorphic response of the balloon-injured rat carotid artery to hormone treatment. Circulation. 95:1301-1307.

23. Hanke, H., et al. 1996. Inhibition of the protective effect of estrogen by progesterone in experimental atherosclerosis. Atherosclerosis. 121:129-138.

24. Grodstein, F., et al. 1996. Postmenopausal estrogen and progestin use and the risk of cardiovascular disease. N. Engl. J. Med. 335:453-461.

25. Hulley, S., et al. 1998. Randomized trial of estrogen plus progestin for secondary prevention of coronary heart disease in postmenopausal women. JAMA. 280:605-613.

26. Carson-Jurica, M.A., Schrader, W.T., and O’Malley, B.W. 1990. Steroid receptor family: structure and functions. Endocr. Rev. 11:201-220.

27. Jenster, G., et al. 1997. Steroid receptor induction of gene transcription: a two-step model. Proc. Natl. Acad. Sci. USA. 94:7879-7884.

28. Tsai, M.J., and O'Malley, B.W. 1994. Molecular mechanisms of action of steroid/thyroid receptor superfamily members. Annu. Rev. Biochem. 63:451-486.

29. King, R.J.B. 1987. Structure and function of steroid receptors. J. Endocrinol. 114:341-349.

30. Beato, M. 1989. Gene regulation by steroid hormones. Cell. 56:335-344.

31. Greene, G.L., and Press, M.F. 1986. I. Steroid receptor structure (including dynamics of the estrogen receptor. J. Steroid Biochem. Mol. Biol. 24:1-7.

32. Lin, A.L., McGill, H.C., Jr., and Shain, S.A. 1982. Hormone receptors of the baboon cardiovascular system. Circ. Res. 50:610-616.

33. Knauthe, R., Diel, P., Hegele-Hartung, C., Engelhaupt, A., and Fritzemeier, K.H. 1996. Sexual dimorphism of steroid hormone receptor messenger ribonucleic acid expression and hormonal regulation in rat vascular tissue. Endocrinology. 137:3220-3227.

34. Ingegno, M.D., et al. 1988. Progesterone receptors in the human heart and great vessels. Lab. Invest. 59:353-356.

35. Sullivan, T.R., Jr., et al. 1995. Estrogen inhibits the response-to-injury in a mouse carotid artery model. J. Clin. Invest. 96:2482-2488.

36. Iafrati, M.D., et al. 1997. Estrogen inhibits the vascular injury response in estrogen receptor $\alpha$-deficient mice. Nat. Med. 3:545-548.

37. Karas, R.H., et al. 1999. Estrogen inhibits the vascular injury response in estrogen receptor $\beta$-deficient female mice. Proc. Natl. Acad. Sci. USA. 96:15133-15136.

38. Lydon, J.P., et al. 1995. Mice lacking progesterone receptor exhibit pleiotropic reproductive abnormalities. Genes Dev. 9:2266-2278.

39. Lydon, J.P., DeMayo, F.J., Conneely, O., and O'Malley, B.W. 1996. Reproductive phenotypes of the progesterone receptor null mutant mouse. $J$. Steroid Biochem. Mol. Biol. 56:67-77.

40. Chappell, P.E., Lydon, J.P., Conneely, O.M., O’Malley, B.W., and Levine, J.E. 2000. Endocrine defects in mice carrying a null mutation for the progesterone receptor gene. Endocrinology. 138:4147-4152.

41. Lindner, V., Fingerle, J., and Reidy, M.A. 1993. Mouse model of arterial injury. Circ. Res. 73:792-796.

42. Sharma, R., and Bulmer, D. 1993. The effects of ovariectomy and subsequent progesterone replacement on the uterus of the pregnant mouse. J. Anat. 137:695-703.
43. Vinijsanun, A., and Martin, L. 1991. Effect of early ovarectomy and steroid hormone replacement of embryo transport, development and implantation in mice. Reprod. Fertil. Dev. 3:35-50.

44. Said, T.K., Conneely, O.M., Medina, D., O’Malley, B.W., and Lydon, J.P. 1997. Progesterone, in addition to estrogen, induces cyclin D1 expression in the murine mammary epithelial cell, in vivo. Endocrinology. 138:3933-3939.

45. Karas, R.H., Patterson, B.L., and Mendelsohn, M.E. 1994. Human vascular smooth muscle cells contain functional estrogen receptor. Circulation. 89:1943-1950.

46. Boyle, E.M., Jr., Lille, S.T., Allair, E., Clowes, A.W., and Verrier, E.D. 1997. Endothelial cell injury in cardiovascular surgery: atherosclerosis. Ann Thorac. Surgery. 63:885-894.

47. Clowes, A.W., Clowes, M.M., Fingerle, J., and Reidy, M.A. 1989. Regulation of smooth muscle cell growth in injured artery. J. Cardiovasc. Pharmacol. 14(Suppl. 6):S12-S15.

48. Morey, A.K., et al. 1998. Oestrogen and progesterone inhibit the stimulated production of endothelin-1. Biochem. J. 330:1097-1105.

49. Shabita, H., et al. 2000. Role of co-activators and co-repressors in the mechanism of steroid/thyroid receptor action. Recent Prog. Horm. Res. 52:141-164.

50. Burris, T.P., Nawaz, Z., Tsai, M.-J., and O’Malley, B.W. 2000. A nuclear hormone receptor-associated protein that inhibits transactivation by the thyroid hormone and retinoic acid receptors. Proc. Natl. Acad. Sci. USA. 92:9525-9529.

51. Power, R.F., Mani, S.K., Codina, J., Conneely, O.M., and O’Malley, B.W. 1991. Dopaminergic and ligand-dependent activation of steroid hormone receptors. Science. 254:1636-1639.

52. Mani, S.K., et al. 1996. Dopamine requires the unoccupied progesterone receptor to induce sexual behavior in mice. Mol. Endocrinol. 10:1728-1737.

53. Neuman, E., et al. 1997. Cyclin D1 stimulation of estrogen receptor transcriptional activity independent of cdk4. Mol. Cell. Biol. 17:5338-5347.

54. Zwijsen, R.M.L., et al. 1997. CDK-independent activation of estrogen receptor by cyclin D1. Cell. 88:405-415.

55. Karas, R.H., Gauer, E.A., Bieber, H.E., Baur, W.E., and Mendelsohn, M.E. 1998. Growth factor activation of the estrogen receptor in vascular cells occurs via a MAP kinase-independent pathway. J. Clin. Invest. 101:2851-2861.

56. Kato, S., et al. 1995. Activation of the estrogen receptor through phosphorylation by mitogen-activated protein kinase. Science. 270:1491-1494.

57. Ignar-Trowbridge, D.M., et al. 1992. Coupling of dual signaling pathways: epidermal growth factor action involves the estrogen receptor. Proc. Natl. Acad. Sci. USA. 89:4658-4662.

58. Ignar-Trowbridge, D.M., Pimentel, M., Parker, M.G., McLachlan, J.A., and Korach, K.S. 1996. Peptide growth factor cross-talk with the estrogen receptor requires the $\mathrm{A} / \mathrm{B}$ domain and occurs independently of protein kinase C or estradiol. Endocrinology. 137:1735-1744.

59. Ignar-Trowbridge, D.M., Pimentel, M., Teng, C.T., Korach, K.S., and McLachlan,J.A. 1995. Cross talk between peptide growth factor and estrogen receptor signaling systems. Environ. Health Perspect. 103(Suppl. 7):35-38

60. Ignar-Trowbridge, D.M., et al. 1993. Peptide growth factors elicit estrogen receptor-dependent transcriptional activation of an estrogenresponsive element. Mol. Endocrinol. 7:992-998. 